\title{
A Game Theoretical Modeling and Simulation Framework For The Integration Of Unmanned Aircraft Systems In To The National Airspace
}

\author{
Negin Musavi* \\ Bilkent University, Ankara, Cankaya 06800, Turkey \\ Kaan Bulut Tekelioglu ${ }^{\dagger}$ \\ Bogazici University, Istanbul, Bebek 34342, Turkey \\ Yildiray Yildiz; Kerem Gunes§, and Deniz Onural ${ }^{\S}$ \\ Bilkent University, Ankara, Cankaya 06800, Turkey
}

\begin{abstract}
The focus of this paper is to present a game theoretical modeling and simulation framework for the integration of Unmanned Aircraft Systems (UAS) into the National Airspace system (NAS). The problem of predicting the outcome of complex scenarios, where UAS and manned air vehicles co-exist, is the research problem of this work. The fundamental gap in the literature in terms of developing models for UAS integration into NAS is that the models of interaction between manned and unmanned vehicles are insufficient. These models are insufficient because a) they assume that human behavior is known a priori and b) they disregard human reaction and decision making process. The contribution of this paper is proposing a realistic modeling and simulation framework that will fill this gap in the literature. The foundations of the proposed modeling method is formed by game theory, which analyzes strategic decision making between intelligent agents, bounded rationality concept, which is based on the fact that humans cannot always make perfect decisions, and reinforcement learning, which is shown to be effective in human behavior in psychology literature. These concepts are used to develop a simulator which can be used to obtain the outcomes of scenarios consisting of UAS, manned vehicles, automation and their interactions. An analysis of the UAS integration is done with a specifically designed scenario for this paper. In the scenario, a UAS equipped with sense and avoid algorithm, moves along a predefined trajectory in a crowded airspace. Then the effect of various system parameters on the safety and performance of the overall system is investigated.
\end{abstract}

\section{Introduction}

Due to their operational capabilities and cost advantages, the interest in Unmanned Aircraft Systems (UAS) is increasing rapidly. However, the UAS industry hasn't realized its potential as much as desired (for example, a fully developed civilian UAS market still does not exist) and the biggest reason behind this is thought to be that UAS still do not have routine access to National Airspace system (NAS). ${ }^{1}$ UAS can fly only in segregated airspace with restricting rules, since technologies, standards and procedures for a safe integration of UAS in airspace haven't matured yet. Aviation industry is very sensitive to risk and for a new vehicle such as UAS to enter into this sector, they need to be proven to be safe and it must be shown that they will not affect the existing airspace system in any negative way. ${ }^{2,3}$ This needs to be done before giving UAS unrestricted access to NAS. Since the routine access of UAS into NAS is not a reality yet and thus there is

*Student, Mechanical Engineering.

†Student, Computer Engineering and Physics.

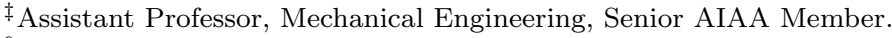

$\S$ Student, Electrical Engineering.

IStudent, Electrical Engineering. 
not enough experience accumulated about the issue, it is extremely hard to see the effects of the technologies and concepts that are developed for the integration. Therefore, employing simulation is currently the only way to understand the effects of UAS integration on air traffic system. ${ }^{4}$ These simulation studies need to be conducted with realistic Hybrid Airspace System (HAS) models, where man and unmanned vehicles co-exist.

Many existing HAS models in the literature are based on the assumption that the pilots of the manned aircraft behave as they should, without making any mistakes. For instance, since manned aviation already implement Traffic Control Alert Systems (TCAS) to avoid collisions, it is assumed that the pilots will always obey TCAS resolution advisory. However, it is not realistic to expect that pilot, as a Decision Maker (DM) of manned aircraft, will always behave deterministically based on the rules or instructions. It is not always predictable whether a pilot agrees with a TCAS resolution advisory or not. ${ }^{5}$ The collision between two aircraft (a DHL Boeing 757 and a Bashkirian Tupolev 154) over Uberlingen, Germany, near the Swiss border On 21:35 (UTC) July 1, 2002, is a good evidence that pilots may decide not to act parallel to TCAS advisory or may ignore traffic controller's commands, during high-stress situations. ${ }^{5}$ In the light of above discussion, it is clear that incorporating human decision making process of pilots in HAS models would improve the predictive power of these models. It is noted that the rules and procedures that need to be followed by the pilots can also be incorporated into the game theoretical modeling framework that is proposed in this paper.

One of the primary impediments that hinders the integration of UAS into NAS is the lack of a matured Sense and Avoid (SAA) capability. Any new SAA should be analyzed in order to determine its potential impacts on surrounding air traffic and specific UAS missions. To perform an analysis and evaluation of any SAA logic, it is necessary to model the actions that a pilot would take facing a conflict. ${ }^{6}$ There are various studies in the literature which utilize HAS models to evaluate the safety of SAA systems. In their work, ${ }^{7}$ Maki et al. constructed a SAA logic based on the model developed by MIT Lincoln Laboratory and Kuchar et $a l^{8}{ }^{8}$ did a rigorous analysis of the TCAS as the SAA algorithm for remotely piloted vehicles. In both of these studies the SAA system is evaluated through simulations in a platform which uses the NAS encounter model developed by the MIT Lincoln Laboratory. In the evaluations, it is assumed that pilot decisions are known a priori and depend on the relative motion of the adversary during a specific conflict scenario and do not take into account the decision process of the pilot. Since, the human decision process is complex and unpredictable this approach is not sufficient to evaluate the performance of a SAA logic. ${ }^{7}$ In addition, these platforms do not evaluate the SAA algorithm in terms of its impact on surrounding air traffic. In their work, Perez-Batlle et al. ${ }^{9}$ classified separation conflicts between manned and unmanned aircraft and proposed separation maneuvers for each class. These maneuvers are tested in simulation environment, where it is assumed that the pilots will follow these maneuvers $100 \%$ of the time without error, which may not be the case in real life scenarios. Florent et al. ${ }^{10}$ developed an SAA algorithm and tested it via simulations and experiments. In both of these tests, it is assumed that the intruding aircraft does not change its path while the UAS is implementing the SAA algorithm. Although this approach is useful for initial testing of the algorithm, not making any move while in a dangerous situation may not fully reflect a pilot's reaction in real life. There are other simulation studies such as ${ }^{11}$ that test and evaluate different collision avoidance algorithms in which human decision making processes are not taken into account and some predefined movements are used as pilot models.

In this study, in order to predict pilot reactions in complex scenarios where UAS and manned aircraft co-exist, in the presence of automation such as SAA system, a game theoretical methodology is employed, which is formally known as semi network-form games (SNFGs). ${ }^{12}$ Using this method, probable outcomes of HAS scenarios are obtained that contains interacting humans (pilots) which also interact with a UAS equipped with SAA algorithms. The obtained pilot model is utilized in close encounters while TCAS and traffic management instructions are up to the pilot to be utilized. To obtain realistic pilot reactions, bounded rationality is imposed by utilizing Level-K approach, ${ }^{13,14}$ a concept in game theory which models human behavior assuming that humans think in different levels of reasoning. In the proposed framework, pilots optimize their trajectories based on a reward function representing their preferences for system states. During the simulations, UAS fly autonomously based on pre-programmed data or flight plan which enables it to execute its mission. In these simulations, the effect of certain system variables, such as horizontal separation requirement and required time to conflict for UAS on the safety and performance of the HAS are analyzed (see ${ }^{15}$ for the importance of these variables for UAS integration.). To enable the UAS to perform autonomously in the simulations, it is assumed that it employs an SAA algorithm.

In prior works, the proposed method was used to investigate small scenarios with 2 interacting humans ${ }^{16-18}$ and medium scale scenarios with 50 interacting pilots. ${ }^{19}$ In the study with 50 pilots, the sim- 
ulation environment utilized a gridded airspace where aircraft moved from a grid intersection to another to represent movement. In addition, the pilots could only observe grid intersections to see whether or not another aircraft was nearby. All these simplifying assumptions decreased the computational cost but also decreased the fidelity of the simulation. In this study, for the first time, a) a dramatically more complex scenario in the presence of manned and unmanned aircraft is investigated, b) the simulation environment is not discretized and the aircraft movements are simulated in continuous time, c) realistic aircraft and UAS physical models are used, d) initial states of aircraft are obtained from real data using Flightradar24 (http://www.flightradar24.com). Hence, a much more representative simulation environment with the inclusion of UAS equipped with an SAA algorithm is used, for the first time.

The organization of the paper is as follows: In Section II, the proposed modeling method is explained. In Section III, the HAS with its components is described in detail. In Section IV, simulation results are provided with detailed discussions. Finally, a conclusion is given in Section V.

\section{Pilot behavior modeling methodology}

The most challenging problem in the prediction of the outcomes of complex scenarios where manned and unmanned aircraft co-exist is obtaining realistic pilot models. A pilot model in this paper refers to a mapping from observations of the pilot to his/her actions. To achieve a realistic human reaction model, certain requirements need to be met. First, the model should not be deterministic since it is known from everyday experience that humans do not always react exactly the same when they are in a given "state". Here, "state" refers to the observations and the memory of the pilot. For instance, observing that an aircraft is approaching from a certain distance is an observation and remembering one's own previous action is memory. Second, pilots should show the characteristics of a strategic DM, meaning that the decisions must be influenced by the expected moves of other "agents". Agents can be either the pilots or automation logic of UAS. Third, the decisions emanating from the model should not always be the best (or mathematically optimal) decisions since it is known that humans behave less than optimal in many situations. Finally, it should be considered that a human DM's predictions about other human DMs are not always correct. To accomplish all of these requirements, a combined level-k reasoning and Reinforcement Learning (RL) method, as a non-equilibrium game theoretical solution concept, is utilized.

\section{A. Game theoretical modeling}

Level-k reasoning is a game theoretical solution concept whose main idea is that humans have various levels of reasoning in their decision making process. Level-0 represents a "non-strategic" DM who does not take into account other DM's possible moves when choosing his/her own actions. This behavior can also be named as reflexive since it only reacts to the immediate observations. An example for a non-strategic decision making can be a pilot choosing to increase aircraft's altitude during a conflict without really considering other aircraft pilot's possible moves. In this study, given a state, a level-0 pilot flies with constant speed and heading starting from its initial position toward its destination. A level-1 DM assumes that the other agents in the scenario are level-0 and takes actions accordingly to maximize his/her rewards. A level-2 DM takes actions as though the other DMs are level-1. In a hierarchical manner, a level-k DM takes actions assuming that other DMs behave as level-(k-1) DMs.

\section{B. Reinforcement learning for the partially observable Markov decision process}

RL is a mathematical learning mechanism which mimics human learning process. Although agents do not really know how the learning task should be achieved, they learn how to interact with the environment. An agent receives an observable message of environment's state, then chooses an action, which changes the environment's state, and environment in return encourages or punishes the agent with a scalar reinforcement signal known as reward. Given a state, when an action increases (decreases) the value of an objective function, which defines the goals of the agent, the probability of taking that action increases (decreases). Since the human DM as the agent in the RL process is not able to observe the whole environment state (i.e. the positions of all of the aircraft in the scenario), the agent receives only partial state information. By assuming that the environment is Markov, and based on the partial observability of the state information, this problem can be generalized to a Partially Observable Markov Decision Process (POMDP). In this study, the RL algorithm developed by Jaakkola et al. ${ }^{20}$ is used to solve this POMDP. In this approach, different 
from conventional RL algorithms, the agent does not need to observe all of the states, for the algorithm to converge. A POMDP value function and Q-value function, in which $m$ and $a$ refer to the observable message of the state, $s$, and action respectively, are given by:

$$
\begin{gathered}
V(m)=\sum_{s \in m} P(s \mid m) V(s) \\
Q(m, a)=\sum_{s \in m} P(s \mid m) Q(s, a) .
\end{gathered}
$$

A recursive Monte-Carlo strategy is used to compute the value function and Q-value function. Policy update is given below, where the policy is updated toward $\pi^{1}$ with $\varepsilon$ learning rate, after each iteration:

$$
\pi(a \mid m) \rightarrow(1-\varepsilon) \pi(a \mid m)+\varepsilon \pi^{1}(a \mid m)
$$

where, $\pi^{1}$ is chosen such that, $J^{\pi^{1}}=\max _{a}\left(Q^{\pi}(m, a)-V^{\pi}(m)\right)$. For any policy $\pi^{1}(a \mid m), J^{\pi^{1}}$ is defined as:

$$
J^{\pi^{1}}=\sum_{a} \pi^{1}(a \mid m)\left(Q^{\pi}(m, a)-V^{\pi}(m)\right)
$$

It is noted that the pilot model, or the policy, is obtained once the policy converges during this iterative process.

\section{Components of the hybrid airspace scenario}

The investigated scenario consists of 180 manned aircraft with predefined desired trajectories and a UAS which moves based on its pre-programmed flight plan from one way-point to another. Fig. 1 shows a snapshot of this scenario where the red squares correspond to manned aircraft and the cyan square corresponds to the UAS. It is noted that the size of the considered airspace is $600 \mathrm{~km} \times 300 \mathrm{~km}$. The space is shown with grids just to make it easier to visually grasp the dimensions (two neighboring grid points are $5 \mathrm{~nm}$ away), otherwise all aircraft, manned or unmanned, moves in the space continuously. Yellow circles show the predetermined waypoints that UAS is required to pass. The blue lines passing through the way-points show the predetermined path of the UAS. It is noted that the UAS does not follow this path exactly since it needs to deviate from its original trajectory to avoid possible conflicts using an on-board SAA algorithm. The initial positions, speeds

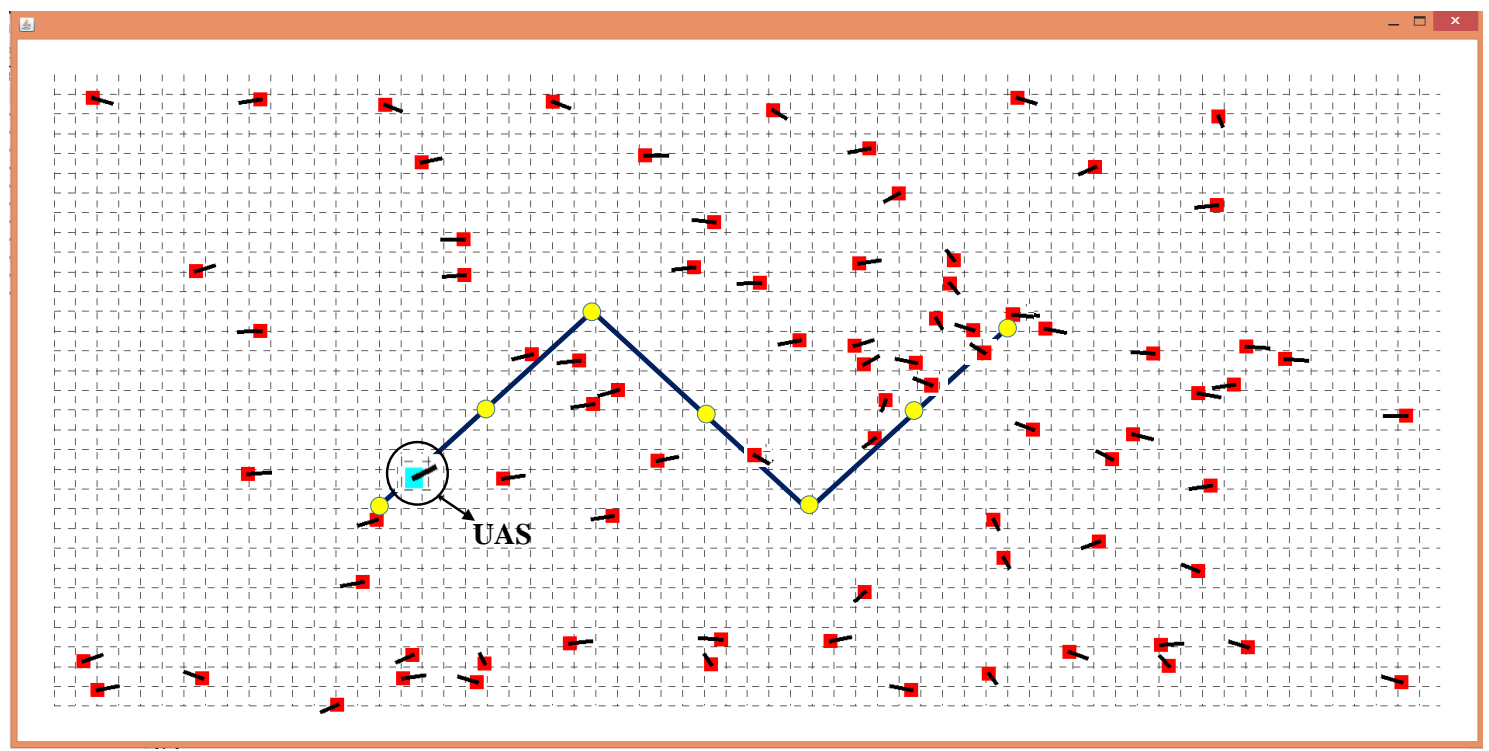

Figure 1: Snapshot of the Hybrid airspace scenario in the simulation platform. Hence, each square stands for a $5 n m \times 5 n m$ area.

and headings of the aircraft are obtained from Flightradar24 website which provides live air traffic data (http://www.flightradar24.com). The data is collected from air traffic on Colorado province, USA airspace. 
It is noted that in the Next Generation Airspace System (NextGen) air travel demand is expected to increase dramatically, thus traffic density is expected to be much more than it is today. To represent this situation, the number of aircraft in the scenario is increased by projecting various aircraft at different altitudes to a given altitude. To handle the increase in aircraft density in NextGen, it is expected that new technologies and automation will be introduced such as automatic dependent surveillance-broadcast (ADS-B), which is a technology that enables aircraft to receive other aircraft identification, position and velocity information and to send its information to others. In the investigated scenario, it is assumed that each aircraft is equipped with ADS-B.

\section{A. Pilot Observations and Memory}

Although ADS-B enables pilots to receive positions and velocities of other aircraft with his/her limited cognitive capabilities, a pilot can not possibly process all information during his/her decision making process. In this study, in order to model pilot limitations, including visual acuity and perception depth as well as limited viewing range of aircraft, it is assumed that the pilots can observe (or process) the information from a limited portion of the airspace around. This limited portion is simulated as equal angular portions of two co-centered circles called the "observation space" which is schematically depicted in Fig. 2. The radius of the first circle represents pilot vision range, which is taken as $1 \mathrm{~nm}$ based on a survey executed in. ${ }^{21}$ The radius of the second circle is a variable that depends on the separation requirements. Since standard separation for manned aviation is $3-5 \mathrm{~nm}^{9}$ this radius is taken as $5 \mathrm{~nm}$. Whenever an intruder aircraft moves toward one of the 6 regions of the observation space (see Fig. 2), pilot perceives that region as "full". Pilot, in addition, can roughly distinguish the approach angle of the approaching intruder. Pilot categorizes a "full" region into four cases; with a) $0^{\circ}<$ approach angle $<90^{\circ}$, b) $90^{\circ}<$ approach angle $<180^{\circ}$, c) $180^{\circ}<$ approach angle $<270^{\circ}$ and d) $270^{\circ}<$ approach angle $<360^{\circ}$. Fig. 2 depicts a typical example, where pilot A observes that aircraft B is moving toward one of the 6 regions that is colored. In this particular example, pilot A perceives the colored region as "full" with approach angle in the interval $\left[90^{\circ}, 180^{\circ}\right]$ and the rest of the regions as "empty". The information about emptiness, fullness of a region and approach angle is fed to the RL algorithm simply by assigning 0 to empty regions and 1, 2, 3, 4 to full regions, based on the approach angle classifications explained above. Pilots also know the best action that would move the aircraft closest to its trajectory (BTA: Best Trajectory Action) and the best action that would move the aircraft closest to its final destination (BDA: Best Destination Action). Moreover, pilots have a memory of what their actions were at the previous time step. Given an observation, the pilots can choose between three actions: $45^{\circ}$ left, straight, or $45^{\circ}$ right, which are coded with numbers 0,1 and 2. Six ADS-B observations, one BTA, one BDA, and one previous move make up nine total inputs for the reinforcement learning algorithm. Observations get 5 values, $0,1,2,3$ or 4 . The previous move, BTA and BDA have three dimensions each: $45^{\circ}$ left, $45^{\circ}$ right, or straight. Therefore, the number of states for which the reinforcement learning algorithm needs to assign appropriate actions is $5^{6} \times 3^{3}=421,875$.

\section{B. Pilot Objective Function}

The goal of the RL algorithm is to find the optimum probability distribution among possible action choices for each state. As explained above, RL achieves this goal by evaluating actions based on their return which is calculated via a reward/objective function. A reward function can be considered as a happiness function, goal function or utility function which represents, mathematically, the preferences of the pilot among different states. In this paper, pilot reward function is taken as

$$
R=w 1 *(-C)+w 2 *(-S)+w 3 *(-C A)+w 4 *(D)+w 5 *(-P)+w 6 *(-E) .
$$

In equation (5), " $C$ " is the number of aircraft within the collision region. Based on the definition provided by the Federal Aviation Administration (FAA), the radius of collision is taken as $500 \mathrm{ft}^{22}$ " $S$ " is the number of air vehicles within the self-separation region. The radius of the self-separation region is $5 \mathrm{~nm} . .^{9}$ " $C A$ " represents whether the aircraft is getting closer to the intruder or going away from the intruder and takes values 1 , for getting closer, or 0 , for going away. " $D$ " represents how much the aircraft gets closer to or goes away from its destination normalized by the maximum distance it can fly in a time step. Time step is determined based on the frequency of pilot decisions. " $P$ " represents how much the aircraft gets closer to or goes away from its ideal trajectory normalized by the maximum distance it can fly in a time step and " $E$ " 


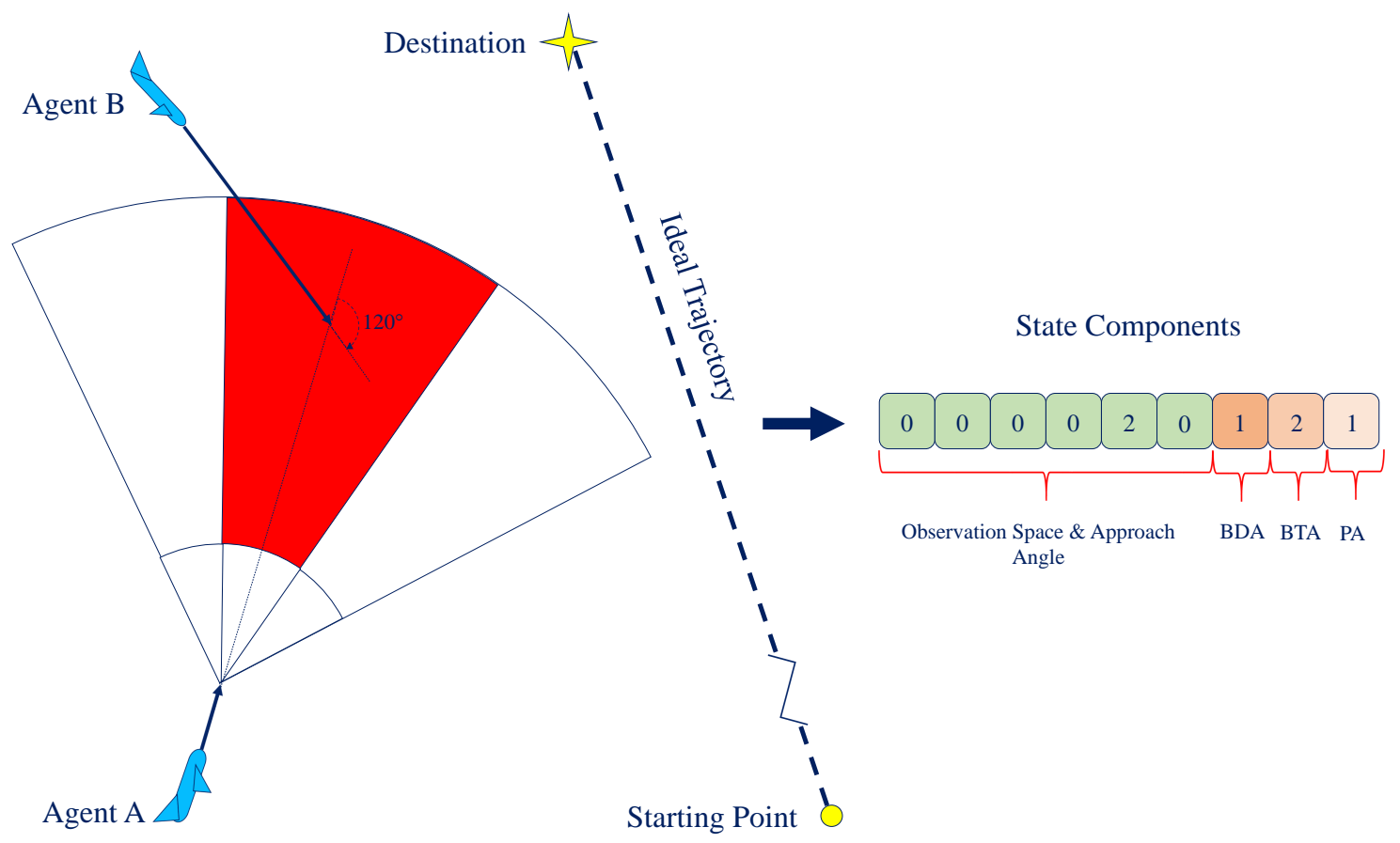

Figure 2: Pilot observation space.

represents whether or not the pilot makes an effort (move). " $E$ " gets a value of 1 if the pilot makes a new move and 0 otherwise.

\section{Manned aircraft model}

The initial position, speed and heading angle of the manned aircraft are obtained from Flightradar24 website (http://www.flightradar24.com). It is assumed that all aircraft are in their en-route phase of travel with constant speed, $\|\vec{V}\|$, in the range of $[150-550]$ knots. Aircraft are controlled by their pilots who can control the heading angle of the aircraft. The pilot may decide to change the heading angle for $45^{\circ},-45^{\circ}$ or may decide to continue with its current direction. Once the pilot gives a heading command, the aircraft moves to the desired heading, $\psi_{d}$, in the constant speed mode. The heading change is modeled by a first order dynamics with the standard rate turn: a turn in which an aircraft changes its heading at a rate of $3^{\circ}$ per second $\left(360^{\circ}\right.$ in 2 minutes) ${ }^{23}$ which is modeled as a first order dynamics with a time constant of $10 \mathrm{~s}$. Therefore, the aircraft heading dynamics can be given as

$$
\dot{\psi}=-\frac{1}{10} \times\left(\psi-\psi_{d}\right)
$$

and the velocity, $\vec{V}=\left(v_{x}, v_{y}\right)$, is then obtained as:

$$
\begin{gathered}
v_{x}=\|\vec{V}\| \sin \psi \\
v_{y}=\|\vec{V}\| \cos \psi .
\end{gathered}
$$

\section{UAS model}

The UAS is assumed to have the dynamics of a RQ-4 Global Hawk with operation speed of $340 \mathrm{knots} .{ }^{24}$ UAS moves according to its pre-programmed flight plan and is also equipped with a SAA system. SAA system can initiate a maneuver to keep UAS away from other traffic, if necessary, by commanding a velocity vector change. Otherwise, UAS will continue moving based on its mission plan. Therefore, UAS always receives a velocity command either to satisfy its mission plan or to protect its safety. Since the UAS has a finite settling time for velocity vector changes, the desired velocity, $\vec{V}_{d}$ cannot be reached instantaneously. Therefore, the 
velocity vector change dynamics of the UAS is modeled by a first order dynamics with a time constant of $1 s^{25}$ which is represented as:

$$
\dot{\vec{V}}=-\left(\vec{V}-\vec{V}_{d}\right)
$$

\section{E. Sense and avoid algorithm}

In order to assure that UAS can detect probable conflicts and can autonomously perform evasive maneuvers, it should be equipped with a SAA system. In this paper, the investigated SAA algorithm is developed by Fasano et al. ${ }^{26}$ The algorithm consist of two phases; conflict detection phase and conflict resolution phase. In the detection phase, the SAA algorithm project the trajectories of the UAS and the intruder aircraft in time, using a predefined time interval, and if the minimum distance between the aircraft during this time is calculated to be less than a minimum required distance, $R$, it is determined that there will be a conflict. In order to prevent the conflict, UAS starts an evasive maneuver in the conflict resolution phase. In the resolution phase of the algorithm, a velocity adjustment is suggested that guarantees minimum deviation from the trajectory. The velocity adjustment command, $\overrightarrow{V_{A}^{d}}$ for UAS is given as

$$
\vec{V}_{A}^{d}=\left[\frac{V_{A B} \cos (\eta-\zeta)}{\sin (\zeta)}\left[\sin (\eta) \frac{\vec{V}_{A B}}{V_{A B}}-\sin (\eta-\zeta) \frac{\vec{r}}{\|\vec{r}\|}\right]\right]+\vec{V}_{B}
$$

where, $\overrightarrow{V_{A}}$ and $\overrightarrow{V_{B}}$ refer to the velocity vectors of the UAS and the intruder. $\vec{r}$ and $\overrightarrow{V_{A B}}$ denote the relative position and velocity between the UAS and the intruder, respectively. $\zeta$ is the angle between $\vec{r}$ and $\overrightarrow{V_{A B}}$ and $\eta$ is calculated as $\eta=\sin ^{-1} \frac{R}{r}$. In the case of multiple conflict detections, the UAS will start an evasive maneuver to resolve the conflict that is predicted to happen earliest.

\section{Simulation results and discussion}

The details of the HAS scenario was explained in section III. In this section, the scenario is simulated to investigate a) the sensitivity of the performance of the SAA algorithm to its parameter variations and b) the effect of the distance and the time horizons on safety and performance of HAS. Since the loss of separation is the most serious issue, the safety metric is taken as the number of separation violations between the UAS and the manned aircraft. Performance metrics, on the other hand, include a) averaged manned aircraft trajectory deviations from their ideal trajectory b) UAS trajectory deviation and c) flight time of the UAS. In all of the simulations, level-0, level-1 and level-2 pilot policies are randomly distributed over the manned aircraft in such a way that $10 \%$ of the pilots fly based on level- 0 policies, $60 \%$ of the pilots act based on level-1 policies and 30\% use level-2 policies. This distribution is based on experimental results discussed in. $^{13}$

\section{A. Sensitivity analyses of the weighting parameters in the objective function}

Before discussing the results of analyzing the SAA algorithm, it's worth analyzing the sensitivity of the pilot model to its parameters. These parameters are the weight vector components in the utility function of pilot, which is given in equation 5. Specifically the effect of the ratio, $r$, of the sum of the weights of the safety components in the utility function over the sum of the weights of the performance components in the utility function, $r=\frac{w 1+w 2+w 3}{w 4+w 5+w 6}$, is investigated, for various traffic densities. The results of this analysis is depicted in Fig. 3. It is seen that as $\mathrm{r}$ increases, the trajectory deviation of both the manned aircraft and the UAS increases, regardless of the traffic density. The cooperation of manned aircraft and the UAS to resolve the conflict reduces the number of separation violations up to a certain value of $r$. However, violations start increasing with a further increase in $\mathrm{r}$. What this means is that, as pilots become more sensitive about their safety and start to overreact to probable conflicts with extreme deviations from their trajectories, the other traffic is effected in a negative way.

\section{B. The effect of distance and time horizon on the performance and safety}

Standard separation distance for manned aviation is $3-5 \mathrm{~nm},{ }^{9}$ however, UAS might require wider separation requirement than manned aviation. In the following analysis, horizontal separation requirement for UAS is called as the distance horizon, and the effect of it is reflected into simulation by assigning this value as the 


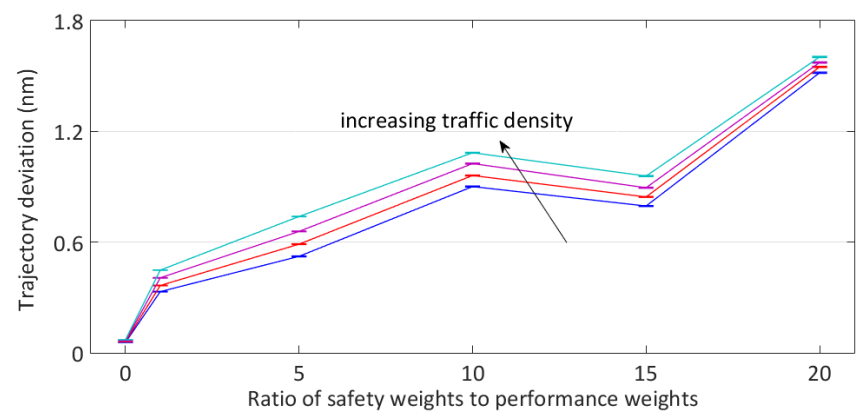

(a) manned aircraft trajectory deviation

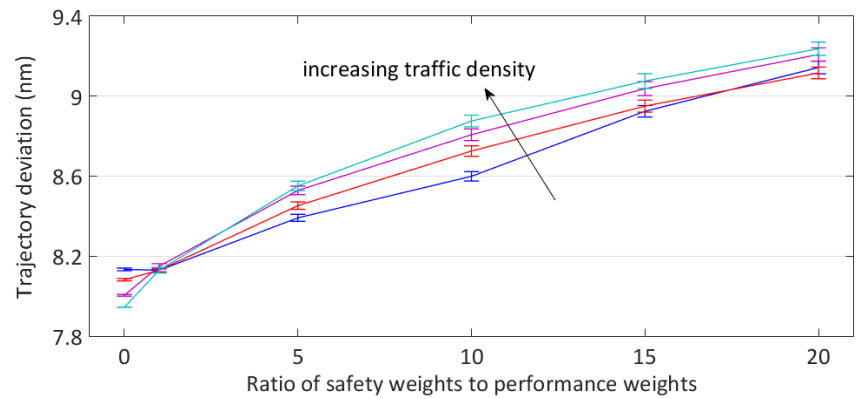

(b) UAS trajectory deviation

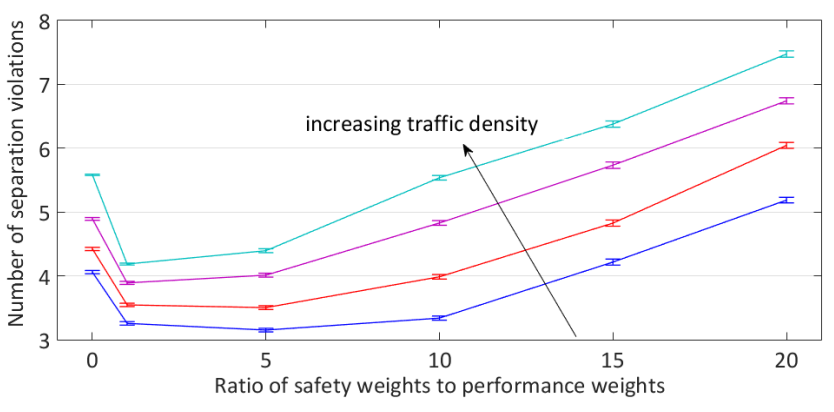

(c) number of separation violations

Figure 3: Pilot aggression and safety vs. performance in HAS. 


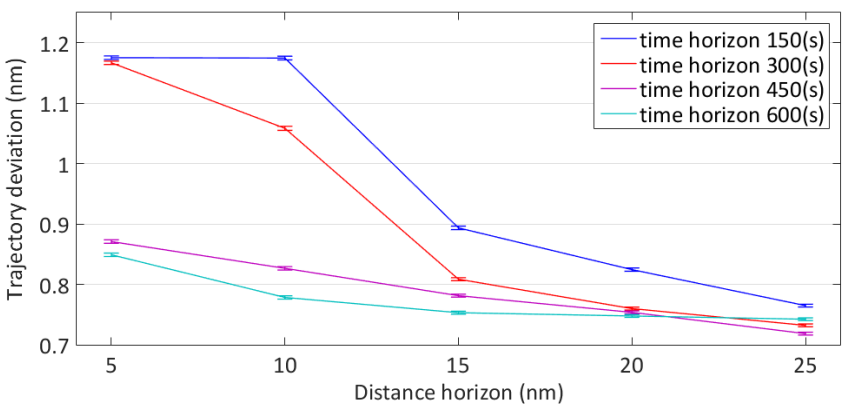

(a) manned aircraft trajectory deviation

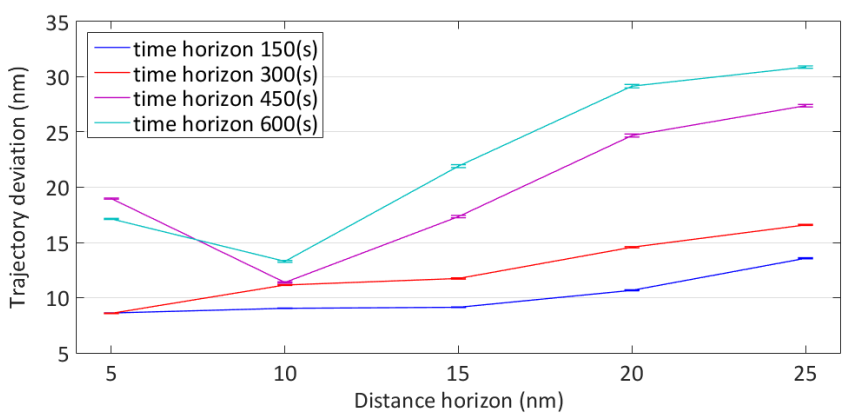

(b) UAS trajectory deviation

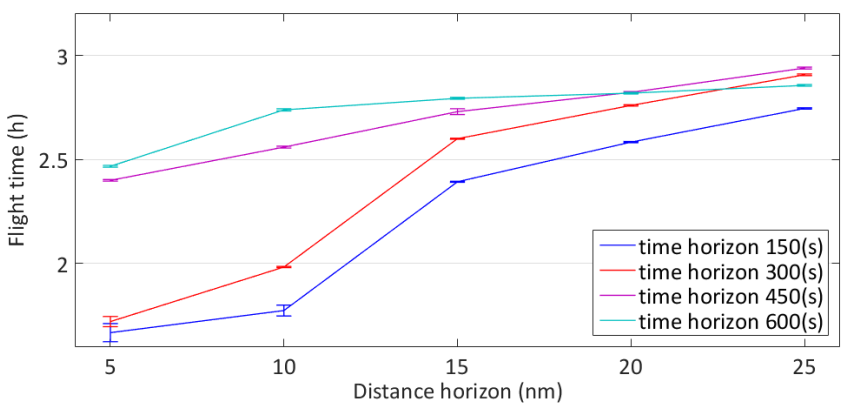

(c) UAS flight time

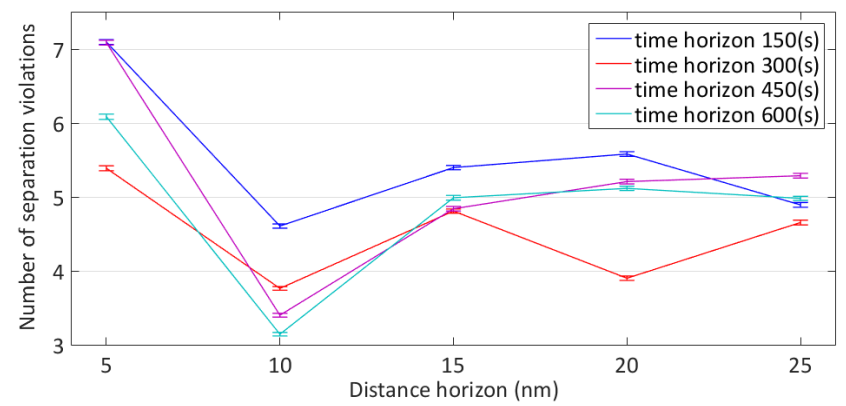

(d) number of separation violations

Figure 4: SAA1 and safety vs. performance in HAS. 
"scan radius" for the SAA algorithm: SAA algorithm considers the aircraft as a possible threat only once the aircraft is within this distance. Another variable whose effect is investigated is defined as the time to separation violation and is called the time horizon from now on. In the simulation, time horizon is used as a time interval that the minimum distance between the aircraft during this time is calculated to be less than horizontal separation requirement of UAS. Figure 4 shows the impact of the time horizon and the distance horizon when SAA algorithm is used. It can be seen from Fig. 4 that by increasing the distance horizon of the UAS, the SAA system detects probable conflicts from a larger distance and the UAS trajectory deviation increases. Increasing the time horizon affects the UAS trajectory deviation similarly. High UAS trajectory deviation results in higher flight times for the UAS to complete its mission. In addition, higher distance and time horizons for the UAS reduces the trajectory deviations of the manned aircraft since handling the conflicts is mostly addressed by the UAS. When UAS foresees the probable conflicts both in terms of time horizon and distance horizon, separation violation generally decreases. Increasing the distance and time horizons after a certain point (10 $\mathrm{nm}$ and $5 \mathrm{~min}$ ) does not improve the safety (number of separation violation), since UAS starts to disturb the traffic unnecessarily due to the overly conservative actions of the SAA system. In this scenario, The required horizontal separation of UAS for a minimal number of separation violations is $10 \mathrm{~nm}$ which is wider than the one in manned aviation. It is noted that this analysis is not enough for a fair analysis of the SAA algorithm since there may be many other factors that need to be taken into account. However, this analysis is another example showing how the proposed game theoretical modeling framework can be utilized.

\section{Conclusion}

In this paper, a game theoretical modeling framework is proposed for use in the integration of Unmanned Aircraft Systems into the National Airspace System. The method provides probabilistic outcomes of complex scenarios where both manned and unmanned aircraft co-exist. Thus, by providing a quantitative analysis, the proposed framework proves itself to be useful in investigating the effect of various system variables, such as horizontal separation distance and time horizon in conflict detection of SAA algorithm, on the safety and performance of the airspace system. The proposed framework is flexible so that any rules and procedures that the pilots are required to follow, for example TCAS advisories, can be incorporated into the model.

\section{Acknowledgment}

We would like to thank the Scientific and Technological Research Council of Turkey (TUBITAK) for its financial support of the project, 3501-114E282, and for its financial support of the student author as the rewardee.

\section{References}

\footnotetext{
${ }^{1}$ Dalamagkidis, V. K. P., Piegl, D. L. A., "On unmanned aircraft systems issues, challanges and operational restrictions preventing integration into national airspace system," Progress in Aerospace Sciences, Vol. 44, No. 7, 2008, pp. 503-519.

${ }^{2}$ Federal Aviation Administration, "Integration of civil unmanned aircraft system (UAS) in the national airspace system (NAS) roadmap," U.S. Department of Transportation, Technical Report, 2013, Available: http://www.faa.gov/about/initiatives/uas-roadmap-2013.pdf.

${ }^{3}$ European RPAS Steering Group, "Roadmap for the integration of civil rpas into the european aviation system," European Comission, Technical Report, 2013, Available:http://ec.europa.eu/enterprise/sectors/aerospace/files/rpas-roadmap-en-pdf.

${ }^{4}$ The MITRE Corporation Center for Advanced Aviation System Developement, "Issues concerning integration of unmanned aerial vehicles in civil airspace," Technical Report, 2014,Available:http://www.mitre.org/sites/default/files/pdf/04-1232.pdf.

${ }^{5}$ Salas, E., and Maurino, D., "Human Factors in Aviation," Elsevier, AcademicPress, 2nd ed., 2010.

${ }^{6}$ Kochenderfer, M. J., Espindle, L. P., Kuchar, J. K., Griffith, J. D., " Correlated Encounter Model for Cooperative Aircraft in the National Airspace System Version 1.0," Lincoln Laboratory, Massachusetts Institute of Technology, 24 October 2008.

${ }^{7}$ Maki, D., Parry, C., Noth, K., Molinario, M., and Miraflor, R.," Dynamic protection zone alerting and pilot maneuver logic for ground based sense and avoid of unmanned aircraft systems," in Proceedings of Infotech@ Aerospace, 2012.

${ }^{8}$ Kuchar, J. K., Andrews, J., Drumm, T. H., Heinz, V., Thompson, S., and Welch, J., "a Safety Analysis process for the traffic alert and collision avoidance system (TCAS) and see-and-avoid systems on remotely poiloted vehicles," in Proceedings of AIAA 3rd Unmanned Unlimitted Technical Conference, Workshop and Exhibit, 2004.

${ }^{9}$ Perez-Batlle, M., Pastor, E., Royo, P., Prats, X., and Barrado, C., "A taxonomy of UAS separation maneuvers and their automated execution," in Proceedings of the 2nd International Conference on Application and Theory of Automation in Command and Control Systems, 2012, pp. 1-11.
} 
${ }^{10}$ Florent. M., Schultz, R. R., and Wang, Z., "Unmanned aircraft systems sense and avoid flight testing utilizing ads-b transceiver," in Proceedings of Infotech@ Aerospace, 2010.

${ }^{11}$ Billingsley. T. B., "Safety analysis of TCAS on global hawk using airspace encounter models,Ph.D. dissertation, Massachusetts Institute of Technology, 2006.

${ }^{12}$ Lee, R., Wolpert, D., "Chapter: Game theoretic modeling of pilot behavior during mid-air encounters." Decision Making with Multiple Imperfect Decision Makers Intelligent Systems Reference Library Series, Springer, 2011.

${ }^{13}$ Costa-Gomez, M. A., Craford, V. P., and Irriberri, N., "Comparing models of strategic thinking in van huyck, battalio, and beil's coordination games," Games and Economic Behavior, vol. 7, no. 2-3, pp,365-376, 1995.

${ }^{14}$ Stahl. D., and Wilson, P., "On players models of other players: Theory and experimental evidence," Games and Economic Behaviorvol. 10, no. 1, pp,218-254, 1995.

15 " Unmanned Aircraft Systems (UAS) Integration in the National Airspace System (NAS) Project," NASA Advisory Council Aeronautics Committee, UAS Subcommittee, June 28, 2012.

${ }^{16}$ Backhaus, S., Bent, R., Bono, J., Lee, R., Tracey, B., Wolpert, D., Xie, D., and Yildiz, Y., "Cyber-physical security: A game theory model of humans interacting over control systems," IEEE Transactions on Smart Grid, vol. 4, no. 4, pp. 2320-2327, 2013.

${ }^{17}$ Yildiz, Y., Lee, R., and Brat, G., "Using game theoretic models to predict pilot behavior in nextgen merging and landing scenario," in Proc. AIAA Modeling and Simulation Technologies Conference, no. AIAA 2012-4487, Minneapolis, Minnesota, Aug. 2012.

${ }^{18}$ Lee, R., Wolpert, D., Bono, J., Backhaus, S., Bent, R., and Tracy, B., " Counter-factual reinforcement learning: How to model decision-makers and anticipate the future," CoRR, Vol. 37, No. 4, pp. 1335-1343, 2014.

${ }^{19}$ Yildiz, Y., Agogino, A., and Brat, G., "Predicting pilot behavior in medium-scale scenarios using game theory and reinforcement learning" Journal of Guidance, Control, and Dynamics, Vol. abs/1207.0852. 2012.

${ }^{20}$ Jaakkola, T., Satinder, P. S., and Jordan, I., "Reinforceent learning algorithm for partially observable markov decision problems" Advances in Neural Information Processing Systems 7: Proceedings of the 1994 Conference, 1994.

${ }^{21}$ Wolfe, R. C., "NASA ERAST Non-Cooperative Detect, See, and Avoid (DSA) Sensor Study," Modern Technology Solutions Inc., Alexandria, VA, September 2002.

${ }^{22}$ NextGen Concept of Operations 2.0. (2007). Washington, DC: FAA Joint Planning and Development Office. Available: http://www.jpdo.gov/ library/NextGen-v2.0.pdf.

${ }^{23}$ Federal Aviation Administration, " Pilot Handbook of Aeronautical Knowledge (FAA-H-8083-25A)," U.S. Department of Transportation, 2008, Available: (http://www.faa.gov/regulations-policies/handbooks-manuals/aviation/pilothandbook/media/FAA-H-8083-25A.pdf).

${ }^{24}$ Dalamagkidis, K., Valavanis, K. P., Piegl, D. L. A., "On Integrating Unmanned Aircraft Systems into the National Airspace System," Springer, 2009.

${ }^{25}$ Mujumdar, A., Padhi, R., "Reactive Collision Avoidance Using Nonlinear Geometric and Differential Geometric Guidance," Journal of Guidance, Control, And Dynamics, Vol. 34, No. 1, January-February 2011.

${ }^{26}$ Fassano, G., Accardo, D., and Moccia, A., "Multi-sensor based fully autonomous non-cooperative collision avoidance system for unmanned air vehicles", J. of a Aerospace Computing, Information, and Communication, Vol. 5, pp. 338-360, 2008. 